\title{
REFLEXIONES ACERCA \\ DE LA CLASIFICACIÓN DE LAS \\ OBRAS LEXICOGRÁFICAS \\ (SEMI)BILINGÜES \\ PARA EL APRENDIZAJE \\ DE LENGUAS
}

\section{REFLEXÕES SOBRE A CLASSIFICAÇÃO DAS OBRAS LEXICOGRÁFICAS (SEMI)BILÍNGUES PARA A APRENDIZAGEM DE LÍNGUAS}

\author{
REFLECTIONS ON THE CLASSIFICATION OF (SEMI) BILINGUAL LEXICOGRAPHIC WORKS \\ FOR LANGUAGE LEARNING
}

Rejane Bueno*

Universidade Federal da Integração Latino-Americana

\begin{abstract}
RESUMEN: Las obras lexicográficas (semi)bilingües son repertorios híbridos que mezclan características de diccionarios bilingües y monolingües. Por ese motivo, la metalexicografía entiende tales obras lexicográficas como complejas y, bajo esa designación, se insertan diccionarios de características muy diversas, lo que conlleva que esas obras han tenido investigaciones muy escuetas y una producción lexicográfica escasa. Se ha observado que tanto la concepción como también la designación de los diccionarios (semi)bilingües varían mucho, de manera que en ese estudio se los concibe como un nuevo subtipo de obra lexicográfica interlingüe. En ese contexto, los principios clasificatorios que describen tales obras lexicográficas no han sido establecidos de forma satisfactoria, por ello proponemos esa reflexión partiendo de una propuesta taxonómica aplicable a diccionarios impresos. La dificultad en elaborar una propuesta de clasificación para las obras (semi)bilingües radica en aunar criterios lingüísticos, históricos y culturales, además del hecho que las obras que existen presentan sistemáticamente una combinación de rasgos de diferentes diccionarios. Aun así, se defiende la importancia de la elaboración de principios clasificatorios para los diccionarios (semi)bilingües por entender que eso aporta conocimientos que refinan los principios de recopilación de esas obras lexicográficas y culminan en la mejoría de su calidad lingüística y lexicográfica.
\end{abstract}

PALABRAS CLAVE: Lexicografía. Lexicografía Pedagógica. Diccionario(semi)bilingüe. Clasificación de diccionarios.

\footnotetext{
* Es profesora adjunta en la Universidade Federal da Integração Latino-Americana (UNILA). Doctora en Estudios de la Traducción por la Universidade Federal de Santa Catarina (UFSC), máster en Estudios lingüísticos - Lexicografía y Terminología por la Universidade Federaldo Rio Grande do Sul (UFRG) y máster en FilologíaHispánica por el Consejo Superior de Investigaciones Científicas (CSIC-España). Email: buenorejane@gmail.com
} 
RESUMO: As obras lexicográficas (semi)bilingues são repertórios híbridos que mesclam características de dicionários bilíngues e monolíngues. Por esse motivo, a metalexicografia entende tais obras lexicográficas como complexas e sob essa designação inseremse dicionários de características muito diversas, o que traz como consequência que essas obras têm sido muito pouco pesquisadas e produzidas. Observou-se que tanto o conceito como a designação dos dicionários (semi)bilíngues são muito variados, de forma que este estudo os concebe como um novo subtipo de obra lexicográfica interlíngue. Nesse contexto, os princípios classificatórios que descrevem essas obras lexicográficas não foram estabelecidos de forma satisfatória, por isso propomos uma reflexão sobre eles a partir de uma proposta taxonômica aplicável a dicionários impressos. A dificuldade de elaborar uma proposta classificatória para as obras (semi)bilíngues reside na junção de critérios lingüísticos, históricos, culturais, além do fato das obras existentes apresentarem sistematicamente uma combinação de traços de diferentes dicionários. Ainda assim, defende-se a importância da elaboração de princípios classificatórios para os dicionários (semi)bilíngues porque entende-se que isso pode proporcionar conhecimentos que refinam os princípios de recopilação dessas obras lexicográficas e melhoram a sua qualidade linguística e lexicográfica.

PALAVRAS-CHAVE: Lexicografia. Lexicografia Pedagógica. Dicionário (semi)bilíngue. Classificação de dicionários.

ABSTRACT:(Semi)bilingual lexicographic works are hybrid repertoires that merge features of bilingual and monolingual dictionaries. For this reason, metalexicography understands such lexicographic works as complex products and, under this designation, there are dictionaries of very different characteristics, which results in the fact that these works have been very little researched and produced. Both the concept and the designation of (semi)bilingual dictionaries are very varied, so this study conceives them as a new subtype of interlingual lexicographic work. In this context, the classification principles that describe these lexicographic works have not been satisfactorily established. Because of that, we propose a reflection on them from a taxonomic proposal to printed dictionaries. The difficulty of elaborating a classification proposal for (semi)bilingual works lies in the combination of linguistic, historical and cultural criteria, besides the fact that existing works systematically present a combination of traces of different dictionaries. Even so, the importance of the elaboration of classification principles for (semi)bilingual dictionaries is defended because it is understood that it can provide knowledge that refines the principles of the compilation of these lexicographic works and improves their linguistic and lexicographic quality.

KEYWORDS: Lexicography. Pedagogical Lexicography. (Semi)bilingual dictionary. Proposal of dictionary classification.

\section{INTRODUCCIÓN}

Las cuestiones que se proponen en este trabajo forman parte de una investigación más amplia que se desarrolló en Bueno (2019) y que estuvo centrada exclusivamente en repertorios lexicográficos (semi)bilingües impresos ${ }^{1}$. Por tanto, la designación que se utiliza, diccionarios (semi)bilingüe, con el prefijo "semi” entre paréntesis, se remonta a eseestudio.

Las obras lexicográficas (semi)bilingües se conocen tradicionalmente en el ámbito de la Lexicografía hispánica a través de la designación semibilingüe. Sin embargo, proponemos esa forma alternativa,(semi)bilingüe,porque al no haber acuerdo teórico sobre si esos diccionarios se acercan más a las obras bilingües o las monolingües, la mayoría de metalexicógrafos que se dedicaron al estudio de esas obras reconocen que tienen un carácter híbrido. De forma que las diferentes unidades que se utilizan para referirse a las obras lexicográficas(semi)bilingües desvelan, además de cuestiones terminológicas, la problemática conceptual que hay en el entorno de esos diccionarios. En otras palabras, no está suficientemente aclarado desde la metalexicografía si las obras (semi)bilingües se constituyen, de hecho, como obras lexicográficas monolingües, bilingües o ambas a la vez. Autores como SnellHornby (1987) y Battenburg (1991) afirmaron que esos repertorios se parecen más a un diccionario monolingüe, porque, entre

\footnotetext{
${ }^{1}$ Aunque la mayoría de los usuarios de diccionarios para el aprendizaje de lenguas parece preferir utilizar repertorios lexicográficos en formato en línea, la investigación de la que procede ese artículo se centró en diccionarios impresos. Principalmente porque tenía como objetivo proponer alternativas de presentación de informaciones lingüísticas en $\mathrm{D}(\mathrm{S}) \mathrm{B}$ a partir del análisis de las insuficiencias que se han constatado en diferentes obras que se han analizado y que eran todas impresas. Todo ello incita a corroborar nuestra intuición que en el ámbito de la lexicografía hispánica aún son muy escuetas las obras lexicográficas de soporte en línea creadas originalmente como repertorios lexicográficos (semi)bilingües.
} 
otras características, incluyen una definición en la L2. Marello (1996) y James (1994) ${ }^{2}$ señalaron que esos diccionarios se identifican más con los bilingües, ya que aportan un equivalente de traducción a la L1 del usuario. Hartmann y James (2001) y Welker (2008) aseveraron que estos diccionarios son un tipo de obra bilingüe, ya que presentan dos lenguas diferentes en su contenido microestructural y, por ello, el prefijo "semi" estaría mal empleado - supuestode Welker (2008, p. 358). Concordamos con elloporque identificamos un repertorio bilingüe como el que pone dos lenguas en contacto en la microestructura de los artículos lexicográficos, sin embargo, seguimos utilizando el prefijo "semi" entre paréntesis para diferenciar estos diccionarios de los repertorios bilingües por defecto.

Por tanto, el diccionario (semi)bilingüe, en adelante $\mathrm{D}(\mathrm{S}) \mathrm{B}$, es un tipo de obra lexicográfica compleja debido a su carácter híbrido, que mezcla en un mismo repertorio características de obras monolingües y bilingües. Esa hibridación de la obra lexicográfica promueve reflexiones desde la perspectiva teórica, como, por ejemplo, para establecer un concepto de tal repertorio o elaborar una terminología unificada para designarlos, como también desde la práctica lexicográfica de compilación de estos diccionarios, porque conlleva aspectos que involucran la Lexicografía monolingüe y la bilingüe. Además, como un factor que complica ese ámbito de análisis, Bueno y Durão (2018, p. 2266) señalaron que bajo la nomenclatura (semi)bilingüe o diccionario bilingualizado hay obras lexicográficas que tienen perfiles muy diversos.

Por ese motivo, es posible sostener que la teoría y la práctica lexicográfica de obras (semi)bilingües constituyen un ámbito de estudios complejo y de difícil precisión, lo que ha tenido como consecuencia que esos repertorios hayan recibido poca atención de parte de los investigadores, tanto del ámbito metalexicográfico, como también de los lexicógrafos que se dedican a la producción de diccionarios pedagógicos. Además, el D $(S) B$ tiene su origen en tradiciones lexicográficas lejanas a la hispánica, lo que motivó a que no se reconocieran como obras de interés académico y editorial en ese contexto.Por tanto, se puede inferir que la magnitud que involucra la amalgama de tales obras lexicográficas se ve reflejada en las escasas investigaciones sobre esos repertorios, así como también en su insuficiente producción lexicográfica ${ }^{3}$.

Por tanto, este trabajo tiene como objetivo principal reflexionar acerca de algunos supuestos que la clasificación de las obras lexicográficas (semi)bilingües involucra, tanto desde la perspectiva teórica, como también práctica. Para ello, repasamos brevemente algunas cuestiones que atañen al concepto y a la designación de esos repertorios lexicográficos, así como también a su origen.

\section{LA PROBLEMÁTICA EN EL ENTORNO DE LA DESIGNACIÓN Y EL CONCEPTO DE LAS OBRAS LEXICOGRÁFICAS (SEMI)BILINGÜES}

Tradicionalmente, en la metalexicografíaha sido complejo comprender en qué categoría lexicográfica se encajan los D(S)B y cuáles son los rasgos que los caracterizan. Además, ha habido una variación significativa en la designación y en la terminología que concierne a ese tipo de obra.

Uno de los primeros teóricos que debatió el concepto de D(S)B fue Hartmann (1992, p. 63-64), quien lo caracterizó como un diccionario bilingualizado, es decir, como una obra que monolingüe a la que se añaden contenidos en la L1 de su potencial usuario. Por tanto, lo identificó como un género particular de diccionario orientado hacia el usuario. Así, consistía en un nuevo subtipo de obra lexicográfica interlingüística. Ese estudioso caracterizó el $\mathrm{D}(\mathrm{S}) \mathrm{B}$ a partir de sus rasgos híbridos entre el diccionario monolingüe de aprendizaje y el bilingüe para aprendices de L2, principalmente por la inclusión de una equivalencia de traducción de cada

\footnotetext{
${ }^{2}$ Es importante poner de relieve que la literatura metalexicográfica que trata de las obras lexicográficas (semi)bilingües se concentra principalmente en los años 90 , cuando esos repertorios lexicográficos se publicaron, mayoritariamente, como obras lexicográficas comerciales. De acuerdo con Duran y Xatara (2005, p. 47), las ediciones KDictionaries, de la editorial Kernerman, son las primeras obras lexicográficas bilingualizadas que popularizaron el concepto lexicográfico de diccionario (semi)bilingüe. Posteriormente a la década de los 90 son escasas las publicaciones de este tipo de repertorio lexicográfico. Por ello, también son más escuetas las reflexiones metalexicográficas acerca de este tipo de diccionario.

${ }^{3}$ En esa dirección,Werner (2005, p. 80) señaló que la escasez de repertorios (semi)bilingües para el aprendizaje de lenguas es una constante en la Lexicografía Pedagógica. Posteriormente, Werner (2010, p. 650) argumentó que el escaso estudio de obras bilingües para el aprendizaje de lenguas es consecuencia de un postulado que, aunque esté ya teóricamente superado, aún lo respetan muchos los autores, que es la absoluta monolingualidad en la enseñanza de idiomas extranjeros, uno de los motivos por el cual los diccionarios bilingües han sido tradicionalmente rechazados en el proceso de enseñanza/aprendizaje de LE.
} 
entrada en la lengua del usuario, afirmando que hasta aquel momento había estado ignorado en las investigaciones sobre diccionarios.

Para Nakamoto (1995),el D(S)B constituye un nuevo tipo de obra lexicográfica que se queda a medio camino entre el diccionario bilingüe y el monolingüe, ya que presenta definiciones monolingües en la L2 y equivalencias de traducción de las entradas en la L1 del usuario. Además, indicó cuales fueron las designaciones que se utilizaron para nombrar esas obras: bilingualizado, semibilingüe, glosado y traducido. Marello (1996, p. 49), además, defendió que los diccionarios bilingualizados son híbridos y tienen una lengua de uso metalingüístico, a diferencia de la obra bilingüe que solamente presenta los equivalentes de las entradas. Propuso una distinción entre $\mathrm{D}(\mathrm{S}) \mathrm{B}$ y el diccionario bilingualizado, afirmando que el $\mathrm{D}(\mathrm{S}) \mathrm{B}$ ha sido concebido originalmente para tal función y el diccionario bilingualizado es una traducción, una modificación de una obra monolingüe que lo precede.

Sin embargo, en Bueno (2019) se ha observado que en la práctica lexicográfica no se ha aplicado esa diferenciación entre obras bilingualizadas y las concebidas originalmente como (semi)bilingües. Principalmente, porque la mayoría de los diccionarios que se titulan (semi)bilingües son, de hecho, obras lexicográficas adaptadas de un original monolingüe para el aprendizaje de lenguas extranjeras y que están dirigidas a grupos mixtos de usuarios de diferentes lenguas maternas (como, por ejemplo, los diccionarios de la editorial Kernerman). De manera que no presentan cualesquier materiales lingüísticos en la L1 del usuario además de la equivalencia de traducción al idioma para el que fueron bilingualizados. En otras palabras, lo que se concibió como bilingualización en ese tipo de obra lexicográfica se centró en el añadido de una unidad léxica en la L1 del usuario al que se dirige potencialmente al final de cada una de las acepciones de cada entrada.

Otra perspectiva interesante acerca del concepto del D(S)B la aportó Marello (1998, p. 310), quien relacionó esas obras a su origen monolingüe, defendiendo que el $\mathrm{D}(\mathrm{S}) \mathrm{B}$ no puede reemplazar la obra monolingüe,pero sí debe formar parte de una de las etapas del aprendizaje de lenguas. En otras palabras, el $\mathrm{D}(\mathrm{S}) \mathrm{B}$ se configura como una obra lexicográfica que debe utilizarse después del diccionario bilingüe y que le encamina al usuario a la consulta de la obra monolingüe.

En una línea de argumentación contraria a reconocer el D(S)B como una nueva tipología de obra lexicográfica, Duran (2004, p. 122) indicó que esos diccionarios son un subtipo del diccionario bilingüe, así que no se constituyen como nuevas obras lexicográficas y tampoco son diccionarios que les subsiguen a los bilingües. Los nombró Diccionarios bilingües pedagógicos. Sin embargo, no estamos de acuerdo con esa propuesta de designación, porque implicaría admitir que el Diccionario bilingüe pedagógico es un diccionario bilingüe que se caracteriza como pedagógico, de forma que todos los demás diccionarios bilingües no serían pedagógicos, lo que no es correcto, en absoluto. De forma que su propuesta terminológica no permite distinguir el Diccionario bilingüe pedagógico de las otras obras bilingües que puedan utilizarse en la enseñanza de lenguas, ya que la característica pedagógica que tiene es compartida con otros diccionarios bilingües, por lo tanto, es una designación ambigua.

El único autor que debatió la concepción de los D(S)B como obras lexicográficas híbridas fue Welker (2008, p.358-359). Ese autor señaló que la concepción de la obra semibilingüe como diccionario híbrido es demasiado amplia y poco informativa ya que puede haber obras lexicográficas híbridas de otros tipos, como, por ejemplo, la mezcla entre diccionario y enciclopedia, de forma que el término híbrido no aclara de qué mezcla se trata, ni desde la perspectiva terminológica, como también desde la perspectiva conceptual de la caracterización práctica de tales obras. Welker (2008, p. 358) propuso como designación a esas obras lexicográficas "Diccionario monolingüe con traducción", defendiendo que no son semi, sino que son totalmente bilingües, ya que muchas no ofrecen más informaciones en la L1 que solamente los equivalentes.

Sin embargo, es posible plantear que, aunque el término híbrido es poco preciso, generalmente en la Lexicografía cuando se menciona una obra lexicográfica con esa característica comúnmente se asocia ese rasgo a los $\mathrm{D}(\mathrm{S}) \mathrm{B}$. Asimismo, al igual que Welker (2008) estamos de acuerdo con que esas obras son de hecho repertorios lexicográficos bilingües en sentido estricto de una obra que debería poner dos lenguas en contacto no solo a partir de la presencia de equivalentes de traducción, sino de la presencia de otro tipo de informaciones (lingüísticas o no) en la L1. Por tanto, concebir a los D(S)B como obras lexicográficas bilingües, permitiría aceptar la posibilidad de que se incluyeran en esos diccionarios otros contenidos en la L1, además del equivalente de traducción. Esos podrían ser, por ejemplo, informaciones semánticas, pragmáticas o enciclopédicas para apoyar la descodificación o 
informaciones morfológicas y/o sintácticas para apoyar la codificación. Lo importante es que esos contenidos en L1 fueran, de hecho, contrastivos con la L2, de forma a poner de relieve las diferencias entre esos idiomas o a destacar alguna similitud entre tales lenguas.

En tal contexto, nos parece contradictorio que Welker (2008, p.358) haya defendido que los D(S)B son repertorios lexicográficos bilingües y luego haya propuesto una designación que asocia esas obras casi exclusivamente a sus características monolingües (Diccionario Monolingüe con Traducción). Eso refleja un aspecto fundamental en el entorno de las reflexiones acerca de las obras lexicográficas (semi)bilingües: que la designación que tradicionalmente se ha utilizado en esas obras no va de la mano de la problemática que atañe a la complejidad de su concepción que mezcla características de obras lexicográficas monolingües y bilingües. En otras palabras, las designaciones que se han utilizado no espejan las posibles funcionalidades que ese tipo de obra lexicográfica puede llegar a tener y tampoco las dificultades propias del ámbito interlingüístico que son inherentes a las obras lexicográficas bilingües, tal y como es el $\mathrm{D}(\mathrm{S}) \mathrm{B}$.

En Bueno y Durão (2018, p. 2276) se presentó un resumen de las características que los D(S)B que se conocen tradicionalmente suelen tener:

- $\quad$ son diccionarios que presentan simultáneamente definiciones en la L2 y equivalentes de traducción en la L1 del potencial usuario;

- se dirigen a aprendices extranjeros de la L2;

- son obras bilingualizadas a partir de un original monolingüe, en la mayoría de los casos;

- pueden utilizarse como obras monolingües siempre y cuando el metalenguaje se haga en la misma lengua de los lemas.

Así pues, concebir al D(S)B como obra lexicográfica monolingüe, lo que implica - reducir su caracterización únicamente a la presencia del equivalente de traducción en la L1 del potencial usuario como el principal rasgo distintivo de esos repertorios lexicográficos frente a los demás. Además de eso, utilizar designaciones que lo conectan únicamente a sus rasgos monolingües y no a los bilingües, genera las siguientes implicaciones principales:

(I) dificulta concebirlo tipológicamente como obra bilíngüe de carácter pedagógico,

(II) perjudica su identificación como obra lexicográfica bilingüe que puede llegar a tener alto potencial contrastivo entre las lenguas que incluye, $y$

(III) merma las posibilidades de utilizarlo como herramienta pedagógica que realmente fomenta el aprendizaje de lenguas.

Por todos esos planteamientos que se han señalado es posible reflexionar que en el ámbito metalexicográfico que se ciñe a la concepción tipológica observable y a la designación de las obras lexicográficas (semi)bilingües:

a) se utilizan designaciones variadas para identificar al $\mathrm{D}(\mathrm{S}) \mathrm{B}$, por tanto, se infiere que no hay unanimidad terminológica para nombrarlo ${ }^{4}$;

b) las discrepancias que se han observado en las designaciones provienen de las interpretaciones conceptuales que cada autor tiene de estas obras lexicográficas, es decir, si los identifican tipológicamente más con repertorios lexicográficos monolingües o bilingües;

c) la complejidad teórica y práctica que atañe a la caracterización de esos repertorios lexicográficos permite múltiples contornos en torno al concepto de obra (semi)bilingüe. Por tanto, proponemos como un criterio para distinguir entre los tipos de obras lexicográficas (semi)bilingüe diferenciar la fuente de la cual procede el diccionario, a grandes rasgos, separándolos en dos grandes grupos: el $\mathrm{D}(\mathrm{S}) \mathrm{B}$ concebido como una creación original desde la planta lexicográfica y el $\mathrm{D}(\mathrm{S}) \mathrm{B}$ bilingualizado concebido como obra adaptada a partir de otras obras lexicográficas.

${ }^{4}$ Se emplea mayoritariamente el término semibilingüe (tradición metalexicográfica hispánica) y bilingualised(tradición metalexicográfica anglosajona). 
En ese apartado se ha podido observar la complejidad involucrada en delimitar el significado y la designación de las obras lexicográficas (semi)bilingües, posiblemente incrementada por el carácter híbrido de esos repertorios lexicográficos al mezclar características de diccionarios monolingües y bilingües. De ahí, cabría cuestionar ¿por qué eso ocurre? Una de las posibles respuestas puede estar relacionada con el origen de ese tipo de obra lexicográfica, así que tratamos de desvelar algunos supuestos acerca de ello en el siguiente epígrafe.

\section{EL ORIGEN DE LAS OBRAS LEXICOGRÁFICAS PARA EL APRENDIZAJE DE LENGUAS}

Concebimos el D(S)B como una categoría de obra lexicográfica pedagógica que se destina al aprendizaje de lenguas. Por ello, proponemos la reflexión acerca de su origen partiendo de observar el nacimiento de las obras generales para el aprendizaje de lenguas.

Para Moreno Fernández (1998, p.152), la génesis del interés por los diccionarios para estudiantes de lenguas extranjeras se relacionacon la necesidad que hubo de fomentar el aprendizaje de lenguas de modo rápido y eficaz a partir de las dos Guerras mundiales. Ya para Cowie (1999, p.3), las circunstancias para el aparecimiento de obras para el aprendizaje de lenguas han sido potencialmente favorables para los editores de diccionarios de aprendizaje de lenguas por medio de los subsidios ofrecidos por los programas de investigaciones acerca del léxico, que casi siempre partían de la perspectiva y de las necesidades europeas.

Sin tener en cuenta los aspectos comerciales que implicaron el desarrollo de la Lexicografía Pedagógica como un todo, Durão (2011, p. 15-16) relacionó el origen de los primeros diccionarios de aprendizaje con los protodiccionarios sumerios. De acuerdo con esa autora, los diccionarios para el aprendizaje se basaron en losrepertorios léxicos que los escribas de la época elaboraban para que sus alumnos los copiaran y los memorizaran.

Es posible reconocer, por tanto, que hay un carácter pedagógico que motivó el nacimiento de los primeros repertorios lexicográficos para el aprendizaje de lenguas. Eso permite asociar una característica pedagógica al surgimiento de los diccionarios de forma general. De ahí que se puede inferir que el origen de esas obras que actualmente se titulan diccionarios pedagógicos es mucho más antiguo de lo que se puede conjeturar. Todo ello corrobora que el nacimiento y la evolución de la Lexicografía Pedagógica pone de relieve algunas relaciones cercanas posibles entre la Lingüística aplicada y la enseñanza de lenguas, con especial atención a los abordajes metodológicos para la enseñanza y el aprendizaje de lenguas ${ }^{5}$.

Así pues, es posible afirmar que la Lexicografía Pedagógica se configura como un área de la Lexicografía que surge con una naturaleza interdisciplinar con convergencias, principalmente, entre:

a) la Lingüística teórica - que puede aportar una descripción lingüística adecuada del léxico que se incorpora al repertorio lexicográfico,

b) la Lingüística aplicada y la didáctica de lenguas - que pueden proporcionar los principios para describir al usuario del repertorio lexicográfico y el tipo de aprendizaje en el que está insertado ese usuario. Como consecuencia de ello, sus necesidades de aprendizaje y destrezas lingüísticas y no lingüísticas que ese aprendizaje involucra;

c) la Metalexicografía y la Lexicografía práctica - que se encargan de orientar los aspectos relacionados con la calidad lexicográfica del diccionario.

\footnotetext{
${ }^{5}$ En Bueno (2007, p. 28-48), se señaló que hay una estrecha relación entre la presencia del diccionario en el aula (que implica el incremento de las reflexiones metalexicográficas y el aumento de la producción práctica de diccionarios para el aprendizaje) y los cambios en los abordajes para la enseñanza de lenguas. Según esa perspectiva, esos cambios implicaron diversas modificaciones en la visión de lengua y, como consecuencia, en la producción de materiales didácticos para su enseñanza. Todo ello permitió que se atribuyera un nuevo y más prominente lugar a las obras de referencia como el diccionario en el aprendizaje de lenguas.
} 
El nacimiento de los D(S)B, de acuerdo con Hartmann (1994, p. 215), se relacionacon su posible funcionalidad. Para él, ese tipo de repertorios debe funcionar como un "puente" entre el diccionario bilingüe tradicional y el monolingüe de aprendizaje: "Bilingualized dictionaries provide a useful bridge between the traditional bilingual dictionary (still associated with low-level proficiency) and the monolingual dictionary aimed at advanced learners".

Según Marello (1998, p. 292), la obra lexicográfica de Hornby, en la década de 60, es el primer ejemplo de diccionario bilingualizado más cercano a lo que hoy se conoce como $\mathrm{D}(\mathrm{S}) \mathrm{B}$. Esa autora puso de relieve que Hornby no estuvo involucrado en el proceso de bilingualización de las obras ${ }^{6}$ porque defendía el principio pedagógico de que una lengua debería aprenderse por el intermedio de ella propia ${ }^{7}$. Es importante destacar que esa es una de las características generales de las obras bilingualizadas, que los editores del repertorio del cual se origina el $\mathrm{D}(\mathrm{S}) \mathrm{B}$ no participan del proceso de bilingualización del diccionario.

De acuerdo con Laufer y Kimmel (1997, p. 362), en consonancia con Hartmann (1994), los D(S)B se originaron a partir de su probable funcionalidad. Estas autoras señalaron que su surgimiento fue motivado por la idea de crear una combinación ideal entre la utilidad real del diccionario y la capacidad de los usuarios para utilizarlo. Es decir, estos repertorios surgieron porque al ofrecer las informaciones monolingües y bilingües las capacidades de los usuarios se potencian y "un diccionario híbrido que contiene los dos tipos de información parece más apropiado" ${ }^{8}$.

Hartmann y James (2001, s.v.bilingualised dictionary) sostuvieron que el surgimiento de las obras bilingualizadas tiene una historia muy larga y que la obra inicial más conocida de este tipo es la bilingualización de un diccionario de Hornby para el chino, en 1948. Afirmaron que la aparición de esos diccionarios fue motivada por las necesidades de los alumnos y profesores de LE.

Para Duran (2004, p.27), las obras (semi)bilingües para aprendices surgieron dentro del segundo momento del movimiento comunicativo, cuando los teóricos empezaron a reevaluar los principios del abordaje comunicativo y comenzaron a reconocer la relevancia del papel del léxico y de la lengua materna en el aprendizaje de lenguas. Asimismo, Duran (2004, p.54) señaló que el D(S)B surgió como un intento de conciliar las ventajas de los repertorios monolingües y bilingües para superar las críticas que ambos tipos de diccionarios recibieron de la comunidad de enseñanza de lenguas.

En una posición análoga a Duran (2004), Climent de Benito (2008, p. 417) indicó que el propósito de crear ese tipo de repertorio lexicográfico era didáctico: solventar el salto de conocimiento que hay entre los niveles iniciales, intermedios y/o avanzados, defendiendo que hay que considerar estas obras como una categoría lexicográfica prototípica.

A modo de conclusión, es posible reflexionar que la misma complejidad que se relaciona con la designación y la concepción de los $\mathrm{D}(\mathrm{S}) \mathrm{B}$ también se observa en el supuesto y controvertido origen de ese tipo de obra lexicográfica. Sin embargo, sería posible resumir la génesis de las obras (semi)bilingüe partiendo de los siguientes aspectos:

(I) los repertorios lexicográficos para el aprendizaje de lenguas, de forma general, surgen asociados a un interés marcadamente pedagógico, con lo cual es posible relacionar su aparecimiento y desarrollo con los diferentes abordajes metodológicos que se han utilizado en la enseñanza de lenguas a lo largo de la historia;

(II) las primeras bilingualizaciones tienen un origen muy lejano, se ubican en los protodiccionarios sumerios;

(III) el origen del $\mathrm{D}(\mathrm{S}) \mathrm{B}$ se vincula a una doble perspectiva: una funcional: fundamentada en principios lexicográficos cuyo objetivo ha sido aunar características de obras monolingües y bilingües en un nuevo modelo de obra lexicográfica y una didáctica: fundamentada en las nuevas perspectivas para la enseñanza de lenguas en las cuales se revaloró la importancia del aprendizaje del léxico y la presencia de la LM en todo el proceso de aprendizaje de la L2.

${ }^{6}$ From the introductions to these dictionaries, we can see that Hornby was not directly involved in the process of bilingualization.

${ }^{7}$ Hornby was firmly committed to the pedagogical principle that English should be learned through the medium of English.

${ }^{8}$ A hybrid dictionary which contains the two types of information would seem most appropriate. 
Además, podría ponerse de relieve que los $\mathrm{D}(\mathrm{S}) \mathrm{B}$, históricamente, trataron también de responder a demandas editoriales y no necesariamente de cubrir aspectos que emanan directamente del ámbito de la enseñanza/aprendizaje de lenguas, lo que incitó a que las investigaciones al respecto y la producción lexicográfica de ese tipo de obras haya sido perjudicada. Además, se puede plantearque cuando eso ocurre queda poco espacio para reflexiones teóricas sobre la calidad del producto lingüístico y lexicográfico en sí. Por otro lado, los materiales didácticos que parten exclusivamente de la teoría en la que se basan sin tener en cuenta las necesidades que existen en el mercado editorial, así como también en las prácticas pedagógicas, suelen desvincularse de las necesidades del mercado y de las cuestiones prácticas que atañen al uso de materiales didácticos.

En el siguiente apartado discutimos acerca de las diferentes perspectivas a partir de las cuales es posible agrupar obras lexicográficas. Además, presentamos las diversas posibilidades de clasificación de los $\mathrm{D}(\mathrm{S}) \mathrm{B}$.

\section{PRINCIPIOS CLASIFICATORIOS APLICABLES A LAS OBRAS LEXICOGRÁFICAS (SEMI)BILINGÜES}

De acuerdo con Bueno (2019, p. 64), la estructura mínima más o menos prototípica de lo que se conoce comúnmente como un $\mathrm{D}(\mathrm{S}) \mathrm{B}$ es la que presenta el lema en L2 + definición en L2 + equivalente en L1, sin embargo, puede haber otras estructuras de obras lexicográficas (semi)bilingües. Ya sea por la diferencia de los procesos de bilingualización que generan diferentes productos lexicográficos o por la diferencia de concepción de la planta de la obra lexicográfica. Por tanto, en este epígrafe se presenta y se discute la relevancia que hay entre los diferentes tipos de repertorios lexicográficos (semi)bilingües. Además, reflexionamos acerca de cuáles han sido los criterios que la Metalexicografía ha utilizado para organizar las diferentes obras (semi)bilingües.

Partimos de criterios de clasificación de obras lexicográficas que se basan en una propuesta taxonómica, aunque también mencionamos las clasificaciones de esos repertorios que parten de organizaciones tradicionales por sistemas de rasgos. Organizar una clasificación de tipos de obras lexicográficas resulta en una actividad compleja por dos motivos principales:

a) en primer lugar, porque en eso influyen factores lingüísticos, históricos y culturales relacionados con las obras lexicográficas y sus usuarios;

b) en segundo lugar, porque las obras lexicográficas existentes presentan, por lo general, una combinación de rasgos pertenecientes a categorías diferentes.

Desde tal perspectiva, Haensch (1982, p. 95-96) puso de relieve que las clasificaciones de repertorios a partir de criterios aislados solamente permiten acceder a representaciones parciales e imprecisas de las obras como pertenecientes a grupos específicos. Por tanto, sería posible inferir que clasificaciones por sistemas de rasgos pueden no describir de forma tan completa las diferentes posibles categorías lexicográficas.

Por ello, proponemos una clasificación de los D(S)B que se basa en una propuesta taxonómica. De acuerdo con Swanepoel (2003, p. 45), una taxonomía es un sistema de clasificación y descripción de elementos de una determinada categoría. Posteriormente, Bugueño Miranda (2014, p. 219) agregó que una clasificación taxonómica es un sistema que permite separar elementos de un grupo en subgrupos que se excluyen y que no son ambiguos. Por lo tanto, es posible inferir que son clasificaciones menos amplias y más específicas que aquellas por sistemas de rasgos.

La importancia de la elaboración de taxonomías en la Lexicografía se refleja, principalmente, en el perfeccionamiento de los instrumentos lexicográficos que se crean según determinadas características y con funciones claramente delimitadas ${ }^{9}$. En otras palabras, conocer los elementos que pertenecen a una clasificación taxonómica y no a otra, permitiría diseñar y producir repertorios lexicográficos con características específicas y que, por ello, posibilitarían ejecutar de manera optimizada las funciones para las cuales estuviera planificada cada tipo específico de obra lexicográfica.

\footnotetext{
${ }^{9}$ Nos basamos en lo planteado por Bugueño Miranda (2014, p. 215), quien afirmó que clasificar las obras lexicográficas permite diseñar instrumentos que están de acuerdo con determinados patrones y parámetros formales que garantizan su real utilidad.
} 
Bugueño Miranda (2014, p.216-219), quien defendió la clasificación de repertorios lexicográficos a partir de la elaboración de taxonomías, propuso tres grandes grupos de criterios para la clasificar las obras lexicográficas:

a) fenomenológico o impresionista: criterio externo a la obra lexicográfica, como, por ejemplo, el tamaño y la densidad macroestructural;

b) funcional: criterio que se atañe a la función prevista para el repertorio lexicográfico, por ejemplo, la orientación por grupos de usuarios;

c) lingüístico: criterio que se relaciona con las características lingüísticas de la obra, como el número de lenguas o la perspectiva del acto comunicativo.

Hay que poner de relieve la complejidad que radica en la elaboración deuna taxonomía que dé cuenta de la totalidad de obras lexicográficas disponibles, por ello nos limitamos únicamente a reflexionar sobre una taxonomía que se aplique a las obras lexicográficas (semi)bilingües. Hacemos hincapié en describir con más detenimiento los criterios funcionales y lingüísticos de esos repertorios lexicográficos.

Duran (2004, p.59) aseveró que no hay acuerdo sobre el lugar que las obras (semi)bilingües ocupan en la metalexicografía, no hay unanimidad sobre cómo o dónde insertar esas obras en las tipologías que ya existen. Hay autores que creen que el formato y la terminología no son originales, por lo tanto, no las clasifican como un subtipo de obra lexicográfica, sino que directamente las describen o bien como obras bilingües o bien como obras monolingües:

Apesar de Lionel Kernerman (1994) proclamar que o dicionário semibilíngüe é um novo tipo de dicionário, alternativo aos dicionários bilíngüe e monolíngüe, os metalexicógrafos ainda não chegaram a um consenso sobre sua inserção dentro da tipologia de dicionários. Aliás, nem o formato do dicionário nem sua denominação são originais, conforme apontam Nakamoto (1995), Marello (1996) e James (2000)

Sobre la importancia de establecer principios clasificatorios adecuados específicamente a los diccionarios (semi)bilingües, Hartmann (1992, p.68) señaló que eso es importante porque incide principalmente en dos ámbitos que se retroalimentan, la teoría y la práctica lexicográfica:

a) en la teoría lexicográfica: una clasificación permite conocer los diferentes (proto)tipos de diccionarios (semi)bilingüe y eso conlleva una mejoría de la comprensión acerca de sus características y diseño;

b) en la práctica lexicográfica: una clasificación aporta conocimientos acerca de la diversidad de obras lexicográficas de determinado tipo y eso proporciona conocimientos que pueden refinar los principios de recopilación de diccionarios, culminando en mejores productos lexicográficos.

El planteamiento de Hartmann (1992) es fundamental para reflexionar acerca de la necesidad de correspondencia entre la teoría y la práctica lexicográfica, con especial atención a las características específicas de las obras lexicográficas que se estudian. Además, pone en evidencia la influencia que puede llegar a tener en la lexicografía práctica conocer los diferentes tipos de obras lexicográficas. En otras palabras, que eso puede incidir en la mejoría de los repertorios lexicográficos concebidos como objetos editoriales.

En ese contexto, el primer metalexicógrafo que propuso una tipología específica de las obras (semi)bilingües fue James (1994), quien declaró que esos diccionarios pueden tener origen en las obras monolingües de aprendizaje y en las monolingües generales. James (1994, p.194) señaló que el diccionario bilingüe es bidireccional, sirviendo adecuadamente a usuarios de dos lenguas diferentes, a diferencia del bilingualizado, que suele ser monodireccional. James (1994, p. 187), además, reconoció que el diccionario bilingualizado consiste en un género particular de obra lexicográfica híbrida e indicó que puede haber cuatro tipos principales de obras lexicográficas (semi)bilingües y que todasellas tienen una función convergente: el fomento del aprendizaje de lenguas, como se observa en el cuadro que se organiza a continuación: 


\begin{tabular}{|c|c|c|c|c|}
\hline & Tipo de $\mathrm{D}(\mathrm{S}) \mathrm{B}$ & Obra de la que parte & Características $<$ & Funciones \\
\hline 1 & $\begin{array}{c}\text { Diccionarios bilingualizados } \\
\text { para aprendices (bilingualised } \\
\text { learners' dictionary) }\end{array}$ & $\begin{array}{c}\text { diccionario para el } \\
\text { aprendizaje de lenguas }\end{array}$ & $\begin{array}{l}\text { - equivalente de traducción en la L1 } \\
\text { agregado posteriormente a la } \\
\text { definición en L2 para confirmar el } \\
\text { sentido vago de las definiciones. }\end{array}$ & $\begin{array}{l}\text { - función didáctica } \\
\text { de fomentar el } \\
\text { aprendizaje }\end{array}$ \\
\hline 2 & $\begin{array}{l}\text { diccionarios bilingualizados } \\
\text { para la enseñanza } \\
\text { (bilingualised teaching } \\
\text { dictionary) }\end{array}$ & $\begin{array}{l}\text { diccionario monolingüe } \\
\text { general para hablantes } \\
\text { nativos del idioma }\end{array}$ & $\begin{array}{l}\text { - ser útil para el usuario nativo (que no } \\
\text { consulta el equivalente, sino la } \\
\text { definición en L1), } \\
\text { - ser útil para el usuario extranjero } \\
\text { (que consulta primero el equivalente } \\
\text { en L2 y solo consulta la definición para } \\
\text { desambiguar el significado, pues esta } \\
\text { es muy compleja), } \\
\text { - permite adaptaciones del material de } \\
\text { la L2 que se agregue, como, por } \\
\text { ejemplo, simplificar las definiciones, } \\
\text { añadir ejemplos o sentidos a las } \\
\text { definiciones. }\end{array}$ & $\begin{array}{c}\text { - función didáctica } \\
\text { de fomentar el } \\
\text { aprendizaje }\end{array}$ \\
\hline 3 & $\begin{array}{c}\text { diccionarios bilingualizados } \\
\text { para el aprendizaje } \\
\text { (bilingualisedlearningdictionary) }\end{array}$ & $\begin{array}{l}\text { diccionario monolingüe } \\
\text { general para hablantes } \\
\text { nativos del idioma }\end{array}$ & $\begin{array}{l}\text { - lemas que están en la L1 del usuario, } \\
\text { - hay glosas en la L2 que están } \\
\text { aprendiendo con la finalidad de } \\
\text { ayudar al usuario de la L1 a codificar } \\
\text { en la L2. }\end{array}$ & $\begin{array}{c}\text { - función didáctica } \\
\text { de fomentar el } \\
\text { aprendizaje }\end{array}$ \\
\hline 4 & $\begin{array}{l}\text { diccionarios semibilingües } \\
\text { (Semi-bilingualdictionary) }\end{array}$ & $\begin{array}{l}\text { diccionariopara el } \\
\text { aprendizaje de L2 }\end{array}$ & $\begin{array}{l}\text { - las partes en la L1 y en la L2 han sido } \\
\text { creadas por el mismo autor del } \\
\text { diccionario, forman parte de un } \\
\text { mismo proyecto lexicográfico, }\end{array}$ & $\begin{array}{l}\text { - función didáctica } \\
\text { de fomentar el } \\
\text { aprendizaje }\end{array}$ \\
\hline
\end{tabular}

Cuadro 1: La tipología de D(S)B de James (1994)

Fuente: elaboración propia

Así, los tipos de obras 1, 2 y 3 caracterizados en la tipología de obras (semi)bilingües de James (1994) son las llamadas bilingualizadas y tienen en común que el contenido que se incluye en la L1 (un equivalente de traducción) sirve para confirmar los significados de las paráfrasis definidoras que están en la L2 del usuario. Además, tales informaciones se agregan posteriormente a la elaboración inicial del diccionario monolingüe y por otro equipo lexicográfico. Eso puede implicar que tales informaciones tengan un tratamiento lexicográfico diferente en cada una de las lenguas.

Otro punto que hay que poner de relieve es que los tipos 1 y 4 de diccionario se originan de diccionarios para el aprendizaje de lenguas extranjeras, frente a los tipos 2 y 3 , que se bilingualizan a partir de obras lexicográficas generales para hablantes nativos. Por tanto, se espera que los tipos 1 y 4 presenten definiciones en la L2 más cortas, más simplificadas, menos enciclopédicas y con el léxico controlado, a diferencia de los tipos 2 y 3 . Por esa característica, sería posible conjeturar que cuando un usuario extranjero consulta ese tipo de obra lexicográfica es posible que la equivalencia de traducción que se incluye en la L1 sea menos útil para actividades de descodificación, aunque esa afirmación queda pendiente de confirmación.

El tipo de diccionario número 4 sería el (semi)bilingüe por defecto y está más cercano a la obra lexicográfica de ese tipo ideal. En otras palabras, presenta la estructura monolingüe derivada de un original orientado a aprendices extranjeros y, por ello, más 
simplificada y cuyo equivalente de traducción forma parte del proyecto lexicográfico inicial. Eso debería contribuir para que el contenido lingüístico de la información en la L1 esté más integrado a las paráfrasis definidoras en la L2.

Por todo ello, afirmamos que la propuesta de James (1994, p. 196) es un aporte importante a ese ámbito de estudios porque presenta una comprensión diferente del proceso de bilingualización de la obra lexicográfica. Ese autor sostuvo que la bilingualización no debe entenderse como un recurso comercial de creación de obras lexicográficas, sino como un recurso de creación de una nueva tipología de obra lexicográfica, poniendo de relieve el matiz didáctico de las obras bilingualizadas y separándolas en dos grandes grupos:

(I) bilingualizadas a posteriori a partir de obras que la preceden (ya sea para hablantes nativos o para aprendices extranjeros) y

(II) concebidas como creaciones originales (semi)bilingües, que son específicas para un tipo de usuario, cuyos apartados traducidos son redactados por el mismo autor.

Sin embargo, no se han encontrado registros de obras lexicográficas en lengua española del segundo tipo, es decir, concebidas originalmente como (semi)bilingües desde su planta lexicográfica. De esta manera, sería posible admitir que esa clase de diccionarios es aún novedosa en el ámbito de la Lexicografía interlingüe hispánica. Además, se constituiría como un prototipo ideal de esas obras lexicográficas porque permitiría explotar idealmente las características de repertorios monolingües y bilingües desde una perspectiva contrastiva entre las lenguas. Con ello, presentaría una finalidad didáctica de fomentar el aprendizaje de una determinada L2 para un usuario de una L1 específica.

Otro autor que contribuyó con la elaboración de una propuesta para clasificar obras (semi)bilingües fue Climent de Benito (2005, p. 404). Él señaló que para clasificar tales diccionarios es necesario tener en cuenta tres parámetros, principalmente:

a) las fuentes de las cuales proceden;

b) las modificaciones que sufren;

c) los propósitos para los que han sido creados.

De lo que planteó Climent de Benito (2005, p.411), quisiéramos señalar un aspecto importante: que las características propias de este género lexicográfico afectan directamente la definición de Lexicografía Pedagógica por dos motivos, principalmente:

(I) porque la hibridación o los diferentes grados de bilingualización de los diccionarios (semi)bilingües incitan a inferir que la diferencia entre los diccionarios monolingües y bilingües deja de ser tan significativa,

(II) porque la hibridación de tales obras surge por y para finalidades didácticas que involucran el aprendizaje de lenguas.

Si por un lado ese autor pone de relieve algo que está tan latente en la Lexicografía Pedagógica como el surgimiento de los D(S)B a partir de motivaciones didácticas, por el otro, desvela una nueva posibilidad de análisis al señalar que el origen de esas obras lexicográficas afecta el concepto de LP porque diluye los límites conceptuales entre repertorios monolingües y bilingües.

Ese planteamiento es de especial relevancia para las reflexiones que proponemos. Estamos parcialmente de acuerdo con ello, creemos que si de hecho las obras (semi)bilingües hubieran sido objeto de más investigaciones metalexicográficas que fueran capaces de contribuir con su desarrollo teórico y práctico, así como si hubieran recibido más atención editorial, seguramente las distinciones teóricas y las fronteras prácticas que existen entre la elaboración de una obra monolingüe y una obra bilingüe no se caracterizarían como una zona borrosa y de difícil separación. Sin embargo, el panorama de investigación metalexicográfica de ese ámbito es escueto, así como los proyectos editoriales de $\mathrm{D}(\mathrm{S}) \mathrm{B}$, de forma que apenas se ha podido concebir el $\mathrm{D}(\mathrm{S}) \mathrm{B}$ como una nueva categoría de obra lexicográfica.

Por todo lo que se ha presentado y reflexionado hasta aquí, parece haber una dicotomía fundamental en el entorno de la taxonomía de las obras lexicográficas (semi)bilingües: que es la fuente de la cual procede el repertorio, es decir: los diccionarios adaptados de

Forum lingüístic., Florianópolis, v.17,n.3,p.4963-4977, jul./set. 2020. 
otras obras que lo preceden - los diccionarios bilingualizados, frente a los concebidos originalmente desde su planta lexicográfica como obras híbridas - los diccionarios (semi)bilingües.

Así pues, cobra relevancia puntualizar algunas cuestiones acerca de una propuesta taxonómica para obras lexicográficas (semi)bilingües:

(I) las diferentes formas de hibridación de obras para el aprendizaje de lenguas pueden contribuir para que esos procesos se vean como posibles, exequibles y/o sistemáticos para bilingualizar una obra lexicográfica. Estos procesos serán diferentes dependiendo del tipo de obra en que se basen y también según cuál será la finalidad de la obra bilingualizada;

(II) las obras (semi)bilingües concebidas bajo tales premisas teóricas y prácticas pueden configurarse como repertorios lexicográficos en los cuales el aspecto binario que hay entre obra monolingüe y bilingüe deja de sostenerse;

(III) la consciencia de que la hibridación de un repertorio tiene como fin prioritario atender al usuario aprendiz convierte ese proceso en un recurso altamente didáctico, posibilitando que la hibridación de las obras lexicográficas para el aprendizaje se convierta en una característica más de las obras cuya finalidad sea pedagógica.

Además, aunque se admite la posibilidad del D(S)B ser una creación original, esa característica sigue siendo innovadora en la Lexicografía Pedagógica, ya que apenas hay diccionarios de ese tipo que no son obras bilingualizadas. Sin embargo, concebir esas obras no solo como adaptadas, sino también como repertorios originales, dotados de características específicas, permitiría añadir a la concepción de $\mathrm{D}(\mathrm{S}) \mathrm{B}$ pedagógico planteamientos más actuales que proporcionarían explotar diferentes posibilidades lexicográficas y/o didácticas en los diccionarios.

Como un resumen a lo que se ha estado exponiendo a lo largo de este trabajo, presentamos un cuadro que se ha extraído de Bueno (2019, p. 70) y que contribuye para la elaboración de una taxonomía preliminar de las obras lexicográficas (semi)bilingües que puedan encontrarse en el ámbito de la Lexicografía Pedagógica:

Parámetros para una clasificación taxonómica de obras (semi)bilingües

\begin{tabular}{c|c|c}
\hline Concepción delrepertorio & $\begin{array}{c}\text { - Adaptación de una obra } \\
\text { que ya existe }\end{array}$ & - Creación original \\
\hline Origen del repertorio & $\begin{array}{c}\text { - Se basa en una obra para el } \\
\text { aprendizaje de lenguas }\end{array}$ & $\begin{array}{c}\text { - Se basa en un diccionario } \\
\text { general }\end{array}$ \\
\hline Soporte de la obra & - Impresa & - Electrónica \\
\hline Presentación de la información & - Inmediata \\
\hline Grados de bilingualización & ¿Cuáles modificaciones ha sufrido del original del cual procede? \\
\hline \multicolumn{2}{c}{ Cuadro 2: Parámetros para la clasificación taxonómica de obras (semi)bilingües } \\
\end{tabular}

A nuestro parecer, tener en cuenta la concepción del repertorio, su origen, su soporte, cómo se presenta la información (con especial atención a la posición de la L1 y de la L2), así como, el grado de bilingualización y los propósitos para el cual se bilingualiza un repertorio lexicográfico son los aspectos básicos que han de conocerse para poder elaborar taxonomías de obras lexicográficas (semi)bilingües. Todo ello deberá contribuir para que los $\mathrm{D}(\mathrm{S}) \mathrm{B}$ se vean como una nueva tipología de obra lexicográfica interlingüe en la cual cada uno de esos aspectos pueda verse reflejado de forma adecuada y con igual grado de importancia en el diccionario. 
Además, el replanteamiento del D(S)B como categoría lexicográfica puede llegar a poner en tela de juicio la distinción entre obra monolingüe y bilingüe e interferir en la concepción propia de LP. Por ello, abogamos por la necesidad de recopilación de obras (semi)bilingües concebidas originalmente como nuevas creaciones lexicográficas de ese tipo, cuyo proyecto lexicográfico incluya desde el principio las dos lenguas involucradas en el repertorio lexicográfico. Eso garantiría, además, que el aspecto contrastivo de los materiales lingüísticos en las dos lenguas estuviera presente a lo largo de todo el repertorio lexicográfico. Así el concepto de D(S)B cambiaría y no se asociaría únicamente a la inclusión de un somero equivalente de traducción al final del artículo lexicográfico.

\section{A MODO DE CONCLUSIÓN}

Ese estudio que propuso organizar algunas reflexiones preliminares en el entorno de clasificación de las obras lexicográficas (semi)bilingüe, empezó presentando las cuestiones relacionadas con la designación y el concepto de esas obras. Se constató que hay alguna variación en las designaciones y que eso procede de la dificultad de comprender esas obras lexicográficas como una categoría lexicográfica específica, debido, principalmente, a su carácter híbrido, que mezcla aspectos de obras lexicográficas bilingües y monolingües en un mismo repertorio lexicográfico.

Al observar el surgimiento de los $\mathrm{D}(\mathrm{S}) \mathrm{B}$, independientemente del diferente origen que los diversos autores señalaron para esas obras, se observó una constante: su origen vinculado a los intereses pedagógicos del aprendizaje de lenguas. Otro aspecto que favoreció el surgimiento de los $\mathrm{D}(\mathrm{S}) \mathrm{B}$ y que vale la pena señalar es la revaloración del papel de la lengua materna del aprendiz extranjero en el aprendizaje de la L2. Solo cuando se reconoció que la L1 tenía un papel importante en el fomento del aprendizaje de la L2 es que los diccionarios bilingües lograron revitalizarse y, como consecuencia, el nacimiento de nuevas obras interlingües, como el $\mathrm{D}(\mathrm{S}) \mathrm{B}$.

Además, se ha observado que hay una dicotomía fundamental en la clasificación de las obras (semi)bilingües, que es la obra adaptada (bilingualizada), frente a la obra concebida como creación original (semi)bilingüe. Esa dicotomía es muy importante porque permite que el $\mathrm{D}(\mathrm{S}) \mathrm{B}$ se conciba como un diccionario bilingüe que logra ir más allá del diccionario bilingüe como repertorio de equivalentes. Es decir, que se caracterice como una obra lexicográfica de nueva planta que puede llegar a ser un repertorio bilingüe contrastivo.

Por tanto, deseamos contribuir para que se replantee el $\mathrm{D}(\mathrm{S}) \mathrm{B}$ de manera que se lo reconozca como una reconceptualización del diccionario pedagógico a la luz de los planteamientos de Nomdedeu Rull (2017). Así, esperamos que en un futuro cercano se incremente el interés por las reflexiones acerca de las obras (semi)bilingües, ya sea desde los parámetros formales que involucran su elaboración, como en las cuestiones prácticas que atañen a su recopilación. Todo ello, permitirá que el D(S)B se establezca como una categoría específica de obra lexicográfica interlingüe dotada de características específicas.

\section{REFERENCIAS}

BATTENBURG, J. D. English monolingual learner's dictionaries. A user-oriented study. Tubinaga: Max Niemeyer, 1991.

BUENO, R. O desenho da microestrutura em um dicionário monolíngüe de espanhol para estudantes brasileiros. 2007. $221 \mathrm{f}$. Dissertação de Mestrado - Programa de Pós-Graduação em Letras, Universidade Federal do Rio Grande do Sul (UFRGS), Porto Alegre, 2007.

BUENO, R. Diccionario (semi)bilingüe para aprendices brasileños de lengua española: una propuesta de tratamiento contrastivo de equivalencia de traducción de verbos. 2019. Tesis Doctoral - Programa de Pós Graduação em Estudos da Tradução, Universidade Federal de Santa Catarina (UFSC), Florianópolis, 2019.

BUENO, R.; DURÃO, A. B. A. B. Reflexiones preliminares acerca del tratamiento de la equivalencia verbal en un diccionario (semi)bilingüe para el aprendizaje de la lengua española. Domínios de Lingu@gem, Uberlândia/MG: EDUFU, v. 12, n. 4, p. 2265 $2294,2018$.

Forum lingüístic., Florianópolis, v.17,n.3,p.4963-4977, jul./set. 2020. 
BUGUEÑO MIRANDA, F. Da classificação de obras lexicográficas e seus problemas: uma proposta de taxonomia. Alfa, São José do Rio Preto/SP, v. 58, n. 1, p. 215-231, 2014.

CHEN, Y. Studies on bilingualized dictionaries: the user perspective.InternationalJournal of Lexicography, Oxonia/UK, v. 24, n. 2, p. 161-197, 2011.

CLIMENT DE BENITO, J. El uso del diccionario bilingüe castellano/valenciano como instrumento didáctico en los ciclos de enseñanza obligatoria de secundaria y bachillerato. 2005. 1914f. Tesis doctoral - Universidad de Alicante, Alicante, 2005.

CLIMENT DE BENITO, J. Los diccionarios semibilingües: principios y clasificación desde un punto de vista didáctico. In: AZORÍN, D. (org.). Actas del II Congreso Internacional de Lexicografía Hispánica. Alicante: Universidad de Alicante, 2008. p. 417427.

COWIE, T. English dictionaries for foreign learners. A History. Oxford: Clarendon Press, 1999.

CUILIAN, Z. The effectiveness of the bilingualized Dictionary: A Psycholinguistic point of view. Chinese Journal of Applied Linguistics, Beijing/CN, v. 33, n. 5, p. 3-14, 2010.

DURAN, M. Dicionários bilíngues pedagógicos: reflexões, análise e propostas. 2004. 131f. Dissertação (Mestrado) - Instituto de Biociências, Letras e Ciências Exatas, Universidade Estadual Paulista Júlio de Mesquita Filho, São José do Rio Preto, 2004.

DURAN, M.; XATARA, C. M. Dicionários semibilíngues: uma inovação? Revista Estudos Linguísticos, Belo Horizonte, v. 13, n. 1, p. 45-57, 2005.

DURÃO, A. B. A. B. Lembremos das velhas obras lexicográficas para redimensionar o papel da Lexicografia e dos novos dicionários. Cadernos de Tradução, Florianópolis/SC, v. 1, n. 27, p. 11-28, jan.- jun. 2011.

JAMES, G. Towards a Typology of Bilingualised Dictionaries. In: JAMES, G. (ed.). Meeting Points in Language Studies. Hong Kong, 1994. p. 184-196.

HAENSCH, G. et al.La lexicografía: de la lingüística teórica a la lexicografía práctica. Madrid: Gredos, 1982.

HARTMANN, R. R. K. Learner's references: from the monolingual to the bilingual dictionary. In: EURALEX INTERNATIONAL CONGRESS ON LEXICOGRAPHY, 5,. 1992. Euralex 92 Proceedings I-IL Papers. Tampere. Finland: Studia Translatologica, v. 2, 1992. p. 63-70.

HARTMANN, R. R. K. Bilingualised Versions of Learners Dictionaries. FremdsprachenLehren und Lernen, [S.1.], n. 23, p. 206-220, 1994.

HARTMANN, R. R. K.; JAMES, G. Dictionary of lexicography. London: Routledge, 2001.

LAUFER, B. A case for a semi-bilingual dictionary for productive purposes. Kernerman Dictionary News, Tel-Aviv, n. 3, Jul. 1995. Disponible en: http://www.kdictionaries.com/newsletter/kdn3-2.htm. Acceso en: 07 oct. 2016.

LAUFER, B.; HADAR, L. Assessing the effectiveness of monolingual, bilingual, and bilingualised dictionaries in the comprehension and production of new words. The Modern Language Journal,New Jersey, v. 81, n. 2, p. 189-196, 1997.

LAUFER, B.; KIMMEL, M. Bilingualised dictionaries: How learners really use them. System, v. 25, n. 3, p. 361-369, 1997. 
LAUFER, B.; MELAMED, L. Monolingual, Bilingual and "Bilingualised" dictionaries: which are more effective, for What and for Whom? In: MARTIN, W. et al (ed.).EURALEX 1994 Proceedings. Amsterdam: International Congress on Lexicography, 1994. p.565-576.

MARELLO, C. Les différents types de dictionnairesbilingües.In: BÉJOINT, H.; THOIRON, P. Lesdictionnairesbilingües. Louvainla-Neuve: Duculot, 1996. p.31-52.

MARELLO, C. Hornby’s bilingualized dictionaries. International Journal of Lexicography, Oxonia/UK, v. 11, n. 4, p. $292-314$, 1998.

MORENO FERNÁNDEZ, F. Diccionarios para el aprendizaje de lenguas extranjeras.In: PRADO, J.; RUBSTALLER, S. (org.).Tendencias en la investigación lexicográfica del español.Huelva: Universidad de Huelva, 1998. p. 101-115.

NAKAMOTO, K. Monolingual or bilingual, that is not the question: the "bilingualised" dictionary. Kernerman Dictionary News, Tel-Aviv, n. 2, Jan. 1995. Disponible en: http//www.kdictionaries.com/newsletter/kdn2-2.htm. Accesoen: 07 oct. 2016.

NOMDEDEU RULL, Hacia una nueva conceptualización de diccionarios pedagógicos del español. In: RUIZ MIYARES, L. Estudios de Lexicología y Lexicografía. Homenaje a Eloína Miyares Bermúdez.Santiago de Cuba: Ediciones Centro de LingüísticaAplicada, 2017.p. 175-206.

PUJOL, D.; CORRIUS, M; MASNOU, J. Print deferrendbilingualised dictionaries and their implications for effective language learning: a new approch to pedagogical lexicography. International Journal of Lexicography, Oxonia/UK, n. 19, p. 197-215, 2006.

SNELL-HORNBY, M. Towards a learner's bilingual dictionary.In:COWIE, A. P (org.).The dictionary and the language learner.Papers from the Euralex Seminar at the University of Leeds. Tubingen, Niemeyer, 1987. p. 159-170.

SWANEPOEL, P. Dictionary typologies: a pragmatic approach. In: STERCKENBURG, P. (ed.).A practical guide to lexicography. Amsterdam: John Benjamin, 2003. p. 44-69.

TERMIGNONI, S. Bases teórico-metodológicas para para um hiperdicionáriosemibilíngue de expressões idiomáticas italianoportuguês em meio a um ambiente virtual de aprendizagem. 2015. 435f. Tese (Doutorado) - Programa de Pós-Graduação em Letras, Universidade Federal do Rio Grande do Sul (UFRGS), Porto Alegre, 2015.

THUMB, J. Dictionary look-up strategies and the bilingualised learner's dictionary: A Think-aloud Study. Tübingen: Niemeyer, 2004. No paginado.

WELKER, H. A. Panorama da lexicografia pedagógica. Brasília: Thesaurus, 2008.

WERNER, R. Diccionarios bilingües del español y otras lenguas iberrománicas: elementos de teoría lexicográfica para combinaciones específicas de lenguas. In: CASTILLO CARBALLO, M. A.; GARCÍA PLATERO, J. M. (org.). La lexicografía en su dimensión teórica. Málaga: Servicio de Publicaciones de la Universidad de Málaga, 2010. p. 643-656.

WERNER, R El diccionario bilingüe y la enseñanza del español como lengua extranjera. Hispanorama, [S.1.], n. 110, p. 75-84, 2005.

\section{(๑) $(\circledast)$}

